\title{
AN ETL PATTERN FOR LOG CONFIGURATION AND ANALYSIS
}

\author{
Bruno Oliveira ${ }^{1}$, Óscar Oliveira ${ }^{1}$, Telmo Matos ${ }^{1}$, Vasco Santos ${ }^{1}$ and Orlando Belo ${ }^{2}$ \\ ${ }^{1}$ CIICESI, School of Management and Technology, Porto Polytechnic, Felgueiras, Portugal \\ ${ }^{2}$ ALGORITMI R\&D Centre, University of Minho, Campus de Gualtar, Braga, Portugal
}

\begin{abstract}
In many scenarios, such as the ones related to Data Warehousing Extract-Transform-Load (ETL) processes, logging techniques are usually applied for capturing event metrics across system levels for system auditing and system recovery. The diversity of strategies and architectures of the toolset used to support the ETL implementation introduces another layer of complexity, both for system development and audit. Although a valuable system diagnosis resource for the development team, logging is generally underestimated, being used only when the system reveals unexpected behaviours and not to drive the ETL system evolution. We believe that the use of logs for steering ETL development and maintenance can improve significantly global system quality. However, this approach is only effective if flexible and efficient logging systems exist. In this paper, we describe a Log Pattern used in a pattern-oriented approach for ETL systems development, which provides a configurable and flexible component for using to drive ETL development and maintenance phases.
\end{abstract}

\section{KEYWORDS}

Data Warehousing, ETL Systems, Logging, Log Pattern, ETL Patterns, Process Mining, Graph Databases

\section{INTRODUCTION}

Data Warehouses (DW) store massive and integrated data that represents an organizational view of transactional data needed for understanding corporative business activities. Extract-Transform-Load (ETL) (Kimball and Caserta, 2014) is one of the key components of a DW, which is responsible for ensuring data integrity and properties correctness. Such properties represent the foundations for data analysis, meaning that any inadequacy in the ETL system may compromise system capability for supporting decision-making processes. The ETL component is responsible for controlling how data is extracted, cleaned, transformed and loaded according to the DW requirements, usually dealing with huge amounts of data collected from several business sources with their own requirements, technology, and availability.

When dealing with an ETL process development, most designers and software architects plan the work to be done considering, beforehand, the toolset to be used to support the implementation, either choosing existing tools, developing their own or following a programmable approach based often on a set of ad-hoc procedures. This means that the initial plan and prototypes designed are in most cases algorithms developed based on a technical programming language or software architecture. This approach is by nature error-prone, hard to understand and to communicate, limiting the process reusability and the system evolution. Also, most of the initial efforts done using software modelling languages (e.g., Unified Modelling Language ${ }^{1}$ ) are ignored by ETL developers because are not well suited for representing all ETL specificities and are viewed as an extra effort that is hard to reuse at physical phases due to the disparities between the representations. Additionally, the maintenance cycle is also challenging since several problems can occur as more business areas are assimilated by the DW system, or the execution requirements are becoming more demanding. Thus, the system evolution must be considered to follow the natural evolution and growth of the data we need to hand (Mannimo and Zhiping, 2006).

\footnotetext{
${ }^{1}$ http://www.uml.org/
} 
The use of a Pattern-Oriented Approach (POA) was proposed by (Oliveira and Belo, 2012) as a way for simplifying and decoupling process components for ETL development and maintenance. In POA, the most commonly used tasks - designed as patterns - are identified and used as preconfigured components for supporting ETL development. This approach covers all ETL development phases, as follows:

1. Conceptual phase - ETL Conceptual representation based on patterns, usually, using Business Process Model and Notation (BPMN).

2. Logical phase - The conceptual model is enriched using a domain specific language (DSL) to represent the specificities of each pattern.

3. Physical phase - The logical model is translated to physical primitives, representing, at least, a skeleton of the target system with the possibility to be executed by existing ETL tools.

As an integral part of the pattern configuration, ETL metadata can be used to support error handling and logging strategies. Thus, Error Handling (Throwable) and Log patterns can be used as patterns' subsystems, encapsulating all the logic behind error and log handling. These two components represent a crucial resource for capturing ETL metadata, and so monitoring all the process stages and handling unexpected exceptions or errors that can occur. All this contributes to improve or correct the logic of an ETL process (Belo et al., 2017).

Based on the POA for the ETL development, we present in this paper the Log Pattern as a configurable and flexible component, that let creating logging strategies, that, alongside to the traditional system audit and recovery use, allows in conjunction with other technologies to streamline the ETL development and maintenance. With flexible logging mechanisms, several log analysis approaches can be exploited and used to drive the ETL development. As an example, we explored the current development on graph databases, e.g., neo $4 \mathrm{j}^{2}$, used to provide efficient and flexible data analytic tools (even for large datasets) that can be used as new resources for ETL development and maintenance. The rest of this paper is organized as follows. Section 2 presents the concept of pattern and how logging techniques can be encapsulated within the POA. Section 3 presents the use of logging following the POA and how generated data can be used to drive the ETL development and maintenance. Conclusions and future work directions are presented in the last Section.

\section{LOG PATTERN}

Tracking ETL system activities is an important task that can be used for identifying inconsistent states, errors or performance bottlenecks. During the ETL workflow execution, several events related to audit or error scenarios are generated. They are stored using transaction log files that are used for providing useful insights about system behaviour considering a specific time window. Logging is a very important component of every ETL system and can be used for several purposes, namely; maintaining consistency and system reliability, monitoring some tasks for ensuring process quality and performance measures, and for identifying bottlenecks and errors that can influence the ETL process operationally. Additionally, log mechanisms can also support recovery mechanisms for handling critical failures and providing a way to roll back data repositories to a consistent state.

The POA frames the ETL development in three main phases: conceptual, logical and physical (Belo et al., 2014). The result of each phase is an input for the next one, representing an incremental development process for building an ETL system. At the conceptual phase, patterns are represented using the BPMN. Basically, patterns are represented as simple "boxes" (activities) used in a workflow context while a subset of the BPMN constructs is used for expressing data and control flow requirements (El Akkaoui et al., 2012). Figure 1 presents an ETL process conceptual model using the POA approach. As the basis for the presented ETL process, a DW repository built to support the decision-making processes from a Movie Rent Company was used. This conceptual model presents the necessary tasks to migrate Movie data from two relational databases to a target DW Movie dimension. As we can see, the process presented in Figure 1 starts with two Change Data Capture (CDC) patterns used to extract only the data changes from Movie data in each one of the involved data sources. For clarity reasons, the ETL patterns are identified using the "\#” character surrounding their name initials. Next, a BPMN parallel gateway is used to indicates that both patterns are independent of each other, meaning that it can be executed in parallel (if the implementation tool used to allow that).

\footnotetext{
${ }^{2}$ https://neo4j.com/
} 


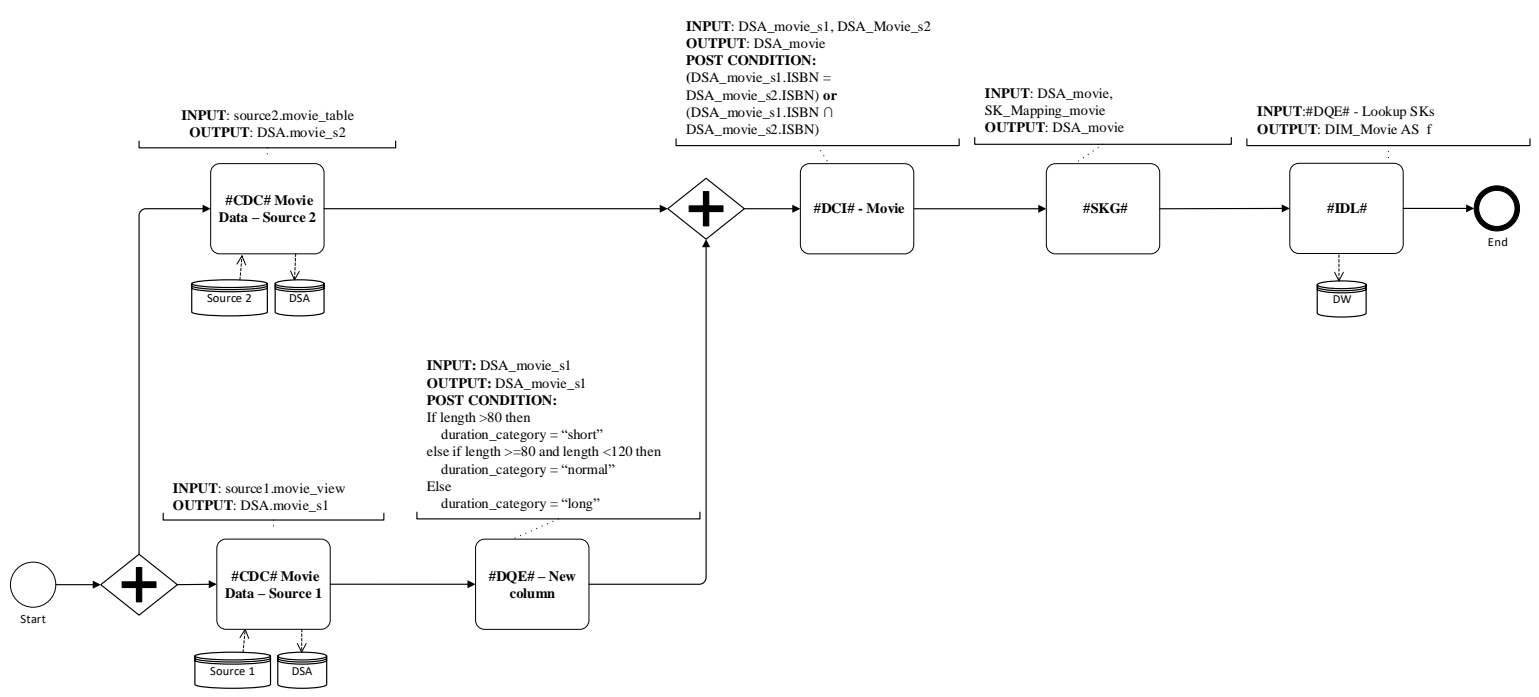

Figure 1. An ETL process used for populating the "Movie" dimension

In some cases, we use BPMN Data Stores artefacts in patterns for improving process readability, representing the type of source that we use - when the data source for a given pattern is the same for the next ones, data source artefacts are omitted. For clarity reasons, we added to each pattern a text annotation artefact, using a specific documentation structure for documenting each BPMN task (Akkaoui et al., 2009). The "input" and "output" keywords (respectively) describe the data handling performed in the data sources (using sourceName.objectName format) for data extraction and load; and "POST CONDITION" keyword for representing the assumptions that should be guaranteed after pattern application. Next, a Data Quality Enhancement (DQE) pattern was used for adding a new column (based on specific rules) that is missing in the Movie's data extracted from Source 1. After data extraction from each data source, the Data Conciliation and Integration (DCI) pattern is used to integrate and consolidate data from the two sources, i.e., for identifying common records existent between the two sources and solve potential conflicts. Next, a Surrogate Key Generator (SKG) pattern is used to generate new surrogate keys for the new records extracted from each data source. Finally, an Intensive Data Loading (IDL) pattern is used to load new data from the Data Staging Area (DSA) to the target DW Movie dimension.

Even in such a simple ETL scenario, logging techniques can be very useful for identifying problems or even possible improvements. Undesirable and unpredictable problems that compromise the system adequacy are often difficult to detect and correct due to a large amount of data involved and to the workflow complexity. Logging is the most used strategy for system monitoring for detecting those problems. As an example, some ETL black points (Belo et al., 2017), which are typical ETL performance bottlenecks, are being analysed using $\operatorname{logs}$ for system efficiency, for reducing system execution time, and decreasing the consumption of computational resources. Additionally, ETL execution usually generates a massive number of logs registering all events in a strict format, i.e., process information and errors with several degrees of severity and detail. Moreover, logs can arrive from diverse sources, each of them with its own format, making it hard to effectively use all this information. This foster the need for flexible logs implementations, as the log information needed at one time, can later be irrelevant or unnecessary. The flexibility of logs can proportionate an alternative use for their use as they can help to drive the ETL development and maintenance.

For enabling a simple log capturing over the process presented in Figure 1, we enrich the conceptual model for providing the logic we need for describing with more detail the behaviour of each pattern, and how to generate logging events as a result of their execution. For that, a domain specific language (Oliveira and Belo, 2015) can be used for reducing ambiguities that are regularly associated with BPMN conceptual models. In Figure 2 we can see a DSL excerpt using JavaScript Object Notation (JSON), representing the configuration applied to the CDC pattern instance represented in the "\#CDC\# Movie Data - Source 1" BPMN task. 


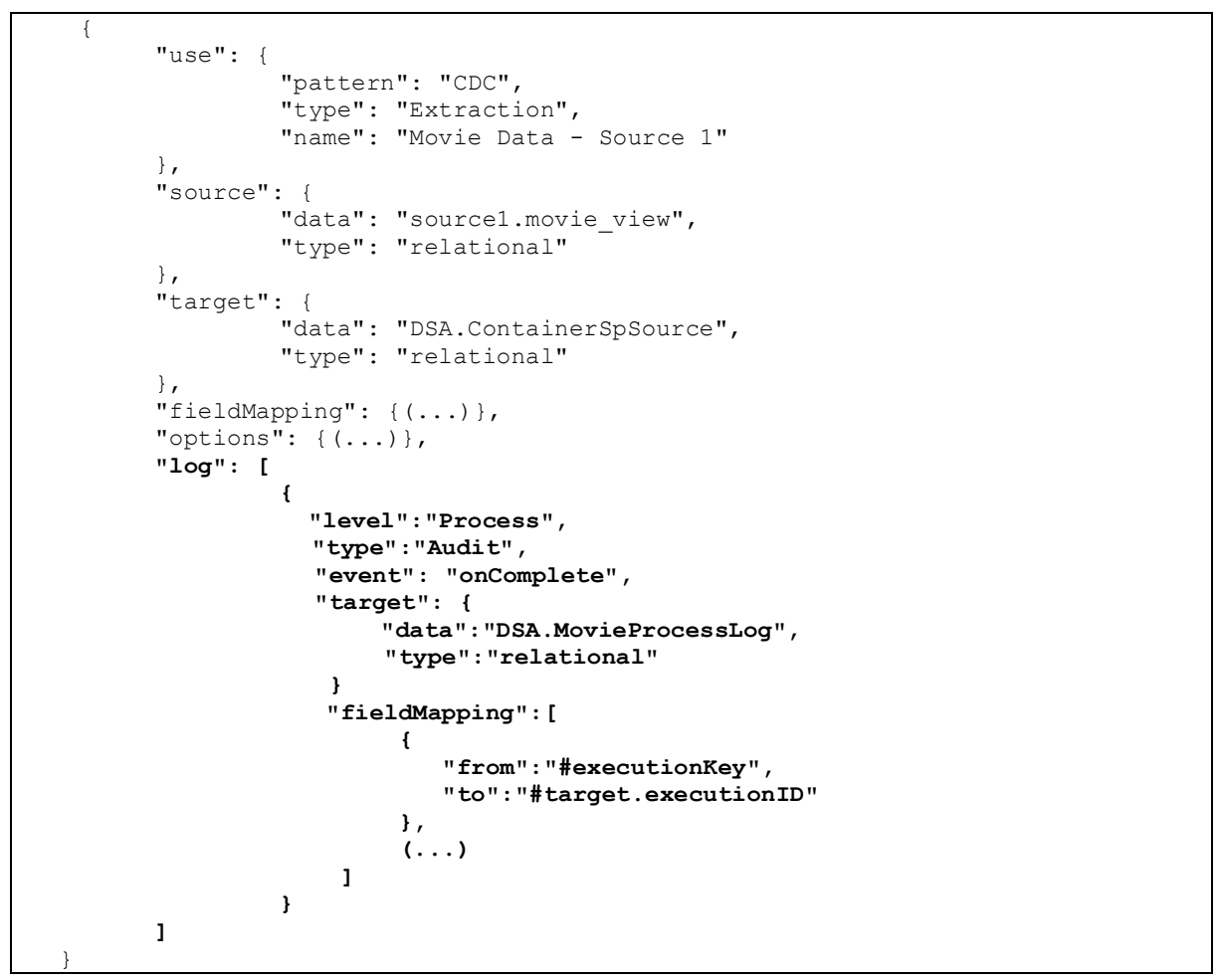

Figure 2. A DSL example of configuring a CDC pattern instance

The DSL covers all the general requirements of each pattern, providing a powerful way to configure its behaviour. The pattern configuration starts with the "USE" keyword followed by the pattern name. Next, a set of blocks delimited by " \{\} " (braces) are used for describing the pattern input ("source"), output ("target"), the field mapping between source and target repositories ("fieldMapping") and the specific configuration properties ("options") that should be applied to each pattern (e.g. for identifying the core metadata used to support the logic for data extraction). We also present a configuration excerpt for the Log pattern component, identifying the rules for capturing the system's events. Several log configurations can be used for the same pattern, which can vary in granularity and scope, triggering the events associated to pattern's internal tasks or by associated to process level like the one presented in the provided example. In any case, several properties can be configured: The "level" property identifies the scope applied to the log activities, which can be "Process", "Pattern" or "Group Pattern" level. The Process level is associated to the scope in which the pattern is used, the Pattern level refers to the pattern's internal activities and Group Pattern level is valid when a set of patterns is placed within a group (which can be identified using BPMN subprocesses);

- The "type" property identifies the log events that should be generated. Basically, a set of $\log$ entries will be generated based on Audit or Throwable events. The Audit type covers audit activities that are automatically registered while the Throwable events cover error/exception scenarios that can occur while ETL is executing.

- The "event" property describes the event that will be used to capture activities. For example, the "onComplete" event is triggered when the pattern completes its execution. Figure 3 presents an example of events categorized in Audit and Throwable.

- The "Target" block allows for describing how log data will be stored. In the example provided in Figure 2, "data" and "type" properties describe the target database table and the type of the log provider used. The "fieldMapping" property describes the log schema using a set of predefined elements that can be used and mapped to the log structure used. Several log elements can be used, e.g., log unique identifier ("Log Key"), activities that originated log events ("Activity") or date fields for identifying the start ("StartDate") and end ("EndDate") dates. The "\#" character is used to refer to predefined or declared elements ("target" prefix is used to refer to the target repository previously declared). 


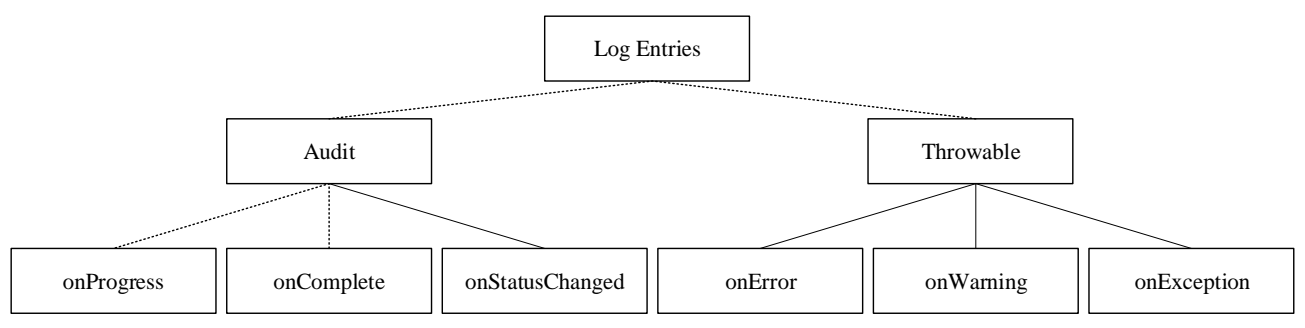

Figure 3. Log events taxonomy according to event nature

The configuration presented in Figure 2 shows some configurations examples for the pattern log component. The rules supporting all these configurations are supported by an extensible meta-model responsible for regulating the POA (Oliveira and Belo, 2016). Assuming that each activity presented in Figure 1 has a similar log configuration to the one presented in Figure 2 (which can also be configured at the process level). Table 1 presents a possible log table (simplified to ease reading) generated based on multiple process executions. To achieve the physical log file, a translation process from the specifications presented should be carried out using a specific ETL tool (Oliveira and Belo, 2018). In this case, the Pentaho Data Integration was used for executing the skeletons generated by a specific parser (the mapping to physical primitives is beyond the scope of this paper).

Table 1. Log table sample data for the process from Figure 1

\begin{tabular}{clcccc}
\hline ExecutionID & \multicolumn{1}{c}{ Activity } & StartDate & Duration & Order $\begin{array}{c}\text { Processed } \\
\text { Records }\end{array}$ \\
\hline 1 & \#CDC\# Movie Data - Source 1 & $2019-02-1211: 10: 54$ & 311 & 1 & $120 \mathrm{~K}$ \\
1 & \#CDC\# Movie Data - Source 2 & $2019-02-1211: 10: 54$ & 261 & 2 & $64 \mathrm{~K}$ \\
1 & \#DQE\# - New column & $2019-02-1211: 16: 05$ & 1212 & 1.1 & $120 \mathrm{~K}$ \\
1 & \#DCI\# - Movie & $2019-02-1211: 36: 17$ & 245 & 3 & $60 \mathrm{~K}$ \\
1 & \#SKG\# & $2019-02-1211: 40: 22$ & 151 & 4 & $60 \mathrm{~K}$ \\
1 & \#IDL\# & $2019-02-1211: 42: 53$ & 210 & 5 & $60 \mathrm{~K}$ \\
$(\ldots)$ & & $(\ldots)$ & $(\ldots)$ & $(\ldots)$ & $(\ldots)$ \\
\hline
\end{tabular}

The configuration of "fieldMapping" property in the "log" block from the Figure 2 configuration, allows for the inclusion of the fields need to build the log file. Additional fields (omitted from Figure 2) were also considered for the log file presented in Table 1, namely:

- The "ExecutionID" column, identifying process execution. Each time the process executes, a new ExecutionID is used;

- The "Activity" column, identifying the activity (a pattern or a pattern internal task) that triggered the event;

- The "StartDate" and "Duration" columns for storing the start date and execution time in seconds for a given activity;

- The "Order" column, representing the position for each log entry activity within the process context, which can be used for ETL debugging. The values for "Order" field are organized hierarchically;

- The "ProcessedRecords" column, identifying the number of records processed by a given activity.

With the log catalogues generated by the pattern log component, ETL events timeline and data lineage can be identified, allowing for the detection of bottlenecks, execution errors, among other things. Thus, the ETL process can be analysed to identify design flaws, providing a way to apply strategies for minimizing process problems and consequently improve data quality and reduce the ETL resources that usually are needed for subsequent data loads. 


\section{ETL LOGGING ANALYSIS}

Nowadays the volume of event log generated from business processes is becoming increasingly high. Although being a valuable resource, this information is often underestimated. Log data has been used in many scenarios to discover several problems in process mining, leading to the system overall performance. In (Uskenbayeva et al., 2015), structured event logs were proposed to build new business processes models with process mining techniques. More recently (Belo et al., 2017), data generated by the ETL process were used to discover black points, which cause additional processing times. The author proposes the analysis of event logs for helping the system administrator to recognize and eliminate these bottlenecks by means of a process mining approach. In (Rozinat et al., 2009), the authors proposed the enhancement of the behaviour of a multi-agent robotic system through the analyses of the log data collected using a process mining tool ( ProM $^{3}$ ).

Analysis of business process or process mining using a graph-oriented database is a relatively new approach. This technology is growing considerably in popularity and applications such as risk management (Rawat, 2017), fraud detection (Akoglu et al., 2015) or social network analysis (Jensen and Neville, 2003). Graph databases (Ian Robinson, Jim Webber, 2015), supported by graph theory, brings another way to work with data, where several characteristics of the model can be identified, suggesting new ways to improve the overall system architectures (Díaz-Pace et al., 2018; Joishi and Sureka, 2014). Logging is a common strategy for mining events, being able to discover specificities in the workflow process that can compromise the overall system. In our approach, we use a graph-oriented model as a use case for the Log Pattern. An intrinsic advantage in a graph-based modelling for process mining lies on the use of graph mining techniques where identification of frequent subgraphs within graph data sets can be discovered (Kashyap et al., 2016; Washio and Motoda, 2003). Additionally, recent advances in graph databases allow effective analytics on a large volume of highly-related data, where traditional approaches, e.g., raw data or relational database, are not suitable.

Considering the flexibility provided by the Log Pattern (described in section 2), the generated log structures can be used as an object for the application of more oriented process mining techniques, based on specific data analysis requirements. For example, a process mining approach for log analysis using graphs is presented to trace system activities. The graph representation specificities show the flexibility of the presented approach to adapt log files metadata. The generated log pattern can be mapped onto a connected graph, streamlining the ETL development and maintenance. Figure 4 presents a view of the resulting graph after mapping the log file described in Table 1.

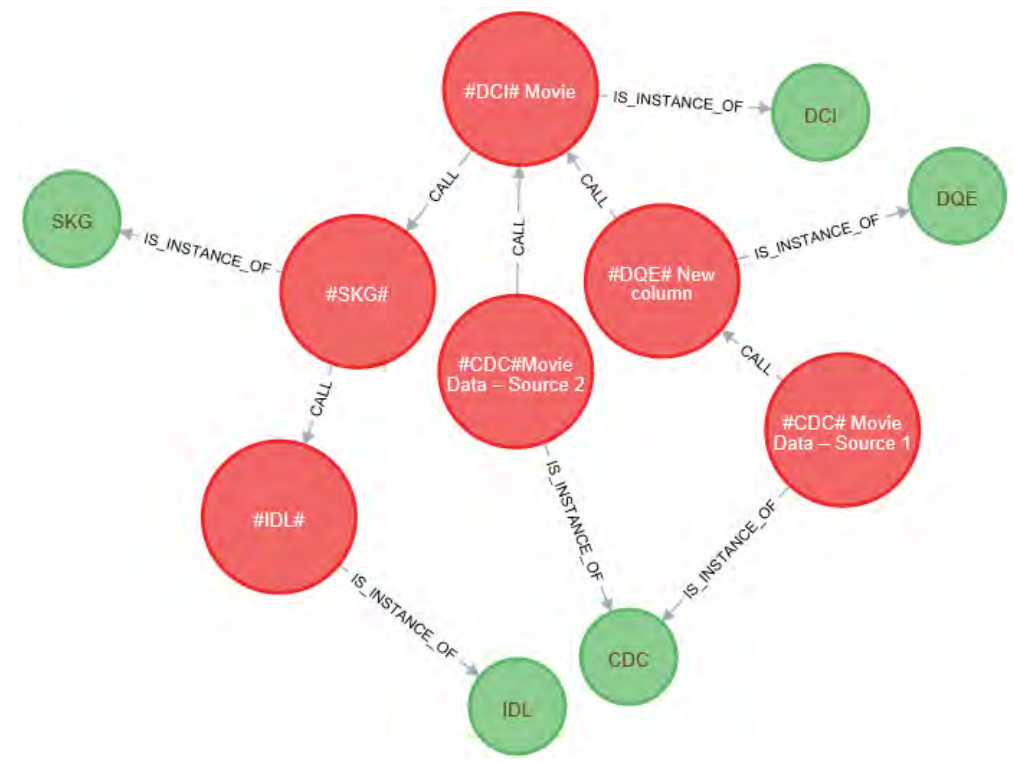

Figure 4. A simple graph model of a log sample data (from Table 1)

\footnotetext{
${ }^{3}$ http://www.promtools.org/doku.php
} 
The conversion from the log file to the presented graph is made identifying the patterns and respective patterns instances. The presented graph has two types of nodes: patterns representing the nature of each activity (green nodes) and the activities used in the BPMN model, representing specific instances of each one of the patterns presented (red nodes). Relations (edges) between nodes are represented as associations and denotes the "IS_INSTANCE_OF" or "CALL" regarding the pattern or the (pattern) instance, respectively.

However, the most complex transformation procedure is related to the activities clustering based on each ETL execution. Basically, the large log data set is grouped in smaller sets of similar data based on the "ExecutionID" column. That way, distinct graphs can be created, allowing the application of data profiling techniques associated with each ETL execution. Figure 4 represents only a single execution, storing its details in each data object using specific properties such as: "ExecutionID", "Duration" and "ProcessedRecords" (extracted from the Table 1). In fact, this is just one among many possibilities to represent a graph using the data provided in the Table 1. Different representations can open different possibilities for new perspectives and insights, which can be adjusted according to analysis requirements.

The analysis of the connected data stored in the graph (Figure 4), allows for understanding anomalies and also for discovering and better perceiving the log data (Chen et al., 2008; Kashyap et al., 2016; Yu, 2018). Such analysis implies data profiling techniques for analysing the structure, contents and log metadata. A series of techniques for collecting statistics and new data insights revealing the process workflow over time, contributing to the understanding of process flaws, identify hidden patterns more easily and potentially improving process performance.

Table 2 presents a node analysis over the pattern instances, representing the ratio between the duration (in seconds) and the number of records processed by the respective pattern (for better reading, we multiplied the values of the ratios by 100). Even for a simple case like the one presented, the ETL designer can analyse and tissue important considerations. Looking at the results, it is possible to observe that the most critical pattern is the "\#DQE\# - New column" with a ratio of 1 . Analysing the ratios and the workflow, we could process the "\#DQE\# - New column" pattern instance after the "\#DCI\# - Movie" pattern, because as there few input records, would reduce the computational time of all ETL system. This workflow restructuration is possible considering that each pattern is decoupled for the remaining ones, i.e., the change in one pattern will not affect the behaviour of the remaining pattern.

Table 2. The ratio between pattern execution duration and the number of processed records

\begin{tabular}{lc}
\hline \multicolumn{1}{c}{ Activity } & Ratio \\
\hline \#CDC\# Movie Data - Source 1 & 0.25 \\
\#CDC\# Movie Data - Source 2 & 0.4 \\
\#DQE\# - New column & 1 \\
\#DCI\# - Movie & 0.2 \\
\#SKG\# & 0.25 \\
\#IDL\# & 0.35 \\
\hline
\end{tabular}

\section{CONCLUSIONS}

In this paper, we presented and discussed a Log pattern for facilitating ETL systems implementation. We can use this pattern component not only at the ETL development phase but also for overcoming well-known problems that usually occur when redesigning an ETL process. We present the Log Pattern under POA that allows for, due to its flexibility, configuring target log structures according to different analytic lenses, improving ETL systems development and maintenance. The graphs application scenario presented, is just an approach for log mining, revealing interesting results that achieved by the Log pattern specification described under a POA approach. The log data mapping to a graph enables the use of graph mining techniques, allowing for the representation of several specificities inherent to graphs modelling. We believe that a flexible approach that can be easily reconfigured and readapted at workflow context makes ETL components more independent each other, which facilitates ETL evolving processes phases that can be used identify undesired situations that can be at least minimized. 
We concluded that besides the discovery of the traditional ETL "black points" that reduce the efficiency of an ETL system, the Log Pattern flexibility provide means to deeper analysis. The next step of this research is to fully exploit the graph modelling advantages to further improve the ETL development and maintenance to attain improved system performance, reliability and quality.

\section{REFERENCES}

Akkaoui, Z. El, Zimanyi, E., El Akkaoui, Z., Zimanyi, E., 2009. Defining ETL worfklows using BPMN and BPEL. Proceeding ACM twelfth Int. Work. Data Warehous. Ol. Dol. 09 41-48.

Akoglu, L., Tong, H., Koutra, D., 2015. Graph based anomaly detection and description: A survey, Data Mining and Knowledge Discovery.

Belo, O., Cuzzocrea, A., Oliveira, B., 2014. Modeling and Supporting ETL Processes via a Pattern-Oriented, Task-Reusable Framework. In: Proceedings - International Conference on Tools with Artificial Intelligence, ICTAI.

Belo, O., Dias, N., Ferreira, C., Pinto, F., 2017. A process mining approach for discovering ETL black points. In: Advances in Intelligent Systems and Computing. pp. 426-435.

Chen, C., Yan, X., Zhu, F., Han, J., Yu, P.S., 2008. Graph OLAP: Towards online analytical processing on graphs. Proc. - IEEE Int. Conf. Data Mining, ICDM 103-112.

Díaz-Pace, J.A., Tommasel, A., Godoy, D., 2018. Can Network Analysis Techniques Help to Predict Design Dependencies? An Initial Study. Proc. - 2018 IEEE 15th Int. Conf. Softw. Archit. Companion, ICSA-C 2018 64-67.

El Akkaoui, Z., Mazón, J.-N.N., Vaisman, A., Zimányi, E., Akkaoui, Z. El, Mazón, J.-N.N., Vaisman, A., Zimányi, E., Zineb El Akkaoui, Mazón, J.-N.N., Vaisman, A., Zimányi, E., Akkaoui, Z. El, Mazón, J.-N.N., Vaisman, A., Zimányi, E., 2012. BPMN-Based Conceptual Modeling of ETL Processes. Lect. Notes Comput. Sci. (including Subser. Lect. Notes Artif. Intell. Lect. Notes Bioinformatics) 7448, 1-14.

Ian Robinson, Jim Webber, E.E., 2015. Graph Databases, Joe Celkos Complete Guide to NoSQL.

Jensen, D., Neville, J., 2003. Data mining in social networks. pp. 287-302.

Joishi, J., Sureka, A., 2014. Performance Comparison and Programming Process Mining Algorithms in Graph-Oriented and Relational Database Query Languages. In: Proceedings of the 19th International Database Engineering \& Applications Symposium on - IDEAS '15. ACM Press, New York, New York, USA, pp. 192-197.

Kashyap, N.K., Pandey, B.K., Mandoria, H.L., Kumar, A., 2016. Graph Mining Using gSpan: Graph-Based Substructure Pattern Mining. Int. J. Appl. Res. Inf. Technol. Comput. 7, 132.

Kimball, R., Caserta, J., 2014. The Data Warehouse ETL Toolkit, Igarss 2014.

Mannimo, M., Zhiping, W., 2006. A framework for data warehouse refresh policies. Decis. Support Syst. 42, $121-143$.

Oliveira, B., Belo, O., 2012. BPMN Patterns for ETL Conceptual Modelling and Validation. 20th Int. Symp. Methodol. Intell. Syst. Lect. Notes Artif. Intell. 7661 LNAI, 445-454.

Oliveira, B., Belo, O., 2015. A domain-specific language for ETL patterns specification in data warehousing systems, Lecture Notes in Computer Science (including subseries Lecture Notes in Artificial Intelligence and Lecture Notes in Bioinformatics).

Oliveira, B., Belo, O., 2016. An Ontology for Describing ETL Patterns Behavior. In: Francalanci, C., Helfert, M. (Eds.), An Ontology for Describing ETL Patterns Behavior. SciTePress, Lisboa, pp. 102-109.

Oliveira, B., Belo, O., 2018. From ETL Conceptual Design to ETL Physical Sketching using Patterns. In: Proceedings of the 20th International Conference on Enterprise Information Systems - Volume 1: ICEIS,. pp. 262-269.

Rawat, D.S., 2017. Analysis of Fraudulent in Graph Data base for Identification \& Prevention. IJIRST -International J. Innov. Res. Sci. Technol. 4, 42-49.

Rozinat, A., Zickler, S., Veloso, M., van der Aalst, W.M.P., McMillen, C., 2009. Analyzing Multi-agent Activity Logs Using Process Mining Techniques. In: Distributed Autonomous Robotic Systems 8. Springer Berlin Heidelberg, Berlin, Heidelberg, pp. 251-260.

Uskenbayeva, R., Kurmangaliyeva, B., Shynybekov, D., Temirbolatova, T., 2015. Application process mining techniques to optimize the business process models based on information systems issuing licenses and permits e-license: Practical research. In: 2015 54th Annual Conference of the Society of Instrument and Control Engineers of Japan (SICE). IEEE, pp. 298-300.

Washio, T., Motoda, H., 2003. State of the art of graph-based data mining. ACM SIGKDD Explor. Newsl. 5, 59.

Yu, F., 2018. Data Profiling: A Holistic View of Data using Neo4j [WWW Document]. F. Eng. Neo4j. URL https://neo4j.com/blog/data-profiling-holistic-view-neo4j/ 